\title{
Synovial fluid monocyte/macrophage subsets and their correlation to patient- reported outcomes in osteoarthritic patients: a cohort study
}

Alejandro Gómez-Aristizábal 1,2,3, Rajiv Gandhi ${ }^{1,2,4}$, Nizar N. Mahomed ${ }^{1,2,4}$, K. Wayne Marshall $^{1,2,4}$ and Sowmya Viswanathan ${ }^{1,2,3,5,6^{*}}$

\begin{abstract}
Background: Chronic, low-grade inflammation of the synovium (synovitis) is a hallmark of osteoarthritis (OA), thus understanding of OA immunobiology, mediated by immune effectors, is of importance. Specifically, monocytes/ macrophages (MФs) are known to be abundantly present in OA joints and involved in OA progression. However, different subsets of OA MФs have not been investigated in detail, especially in terms of their relationship with patient-reported outcome measures (PROMs). We hypothesized that levels of synovial fluid (SF) MФ subsets are indicative of joint function and quality of life in patients with $\mathrm{OA}$, and can therefore serve as biomarkers and therapeutic targets for OA.
\end{abstract}

Methods: In this cohort study, synovial fluid leukocytes (SFLs, $N=86$ ) and peripheral blood mononuclear cells $(n=53)$ from patients with knee OA were characterized. Soluble MФ receptors and chemokine (sCD14, sCD163, CCL2, CX3CL1) levels were detected in SF using immunoassays. Linear models, adjusted for sex, age and body mass index, were used to determine associations between SF MФs and soluble factors with PROMs $(N=83)$. Pearson correlation was calculated to determine correlation between MФ subsets, $T$ cells and soluble factors.

Results: SF MФs were the most abundant SFLs. Within these, the double-positive CD14 $4^{+} C D 16^{+}-M \Phi$ subset is enriched in knee OA SF compared to the circulation. Importantly, MФ subset ratios correlated with PROMs, specially stiffness, function and quality of life. Interestingly, the SF CD14 $4^{+} \mathrm{CD} 16^{+}-\mathrm{MD}$ subset ratio correlated with SF chemokine (C-C motif) ligand 2 (CCL2) levels but not with levels of SCD163 or SCD14; we found no association between PROMs and either SF CCL2, sCD163, sCD14 or CX3CL1 (which was below detection levels). All SF MФs displayed high levels of HLA-DR, suggesting an activated phenotype. Correlation between OA SF MФ subsets and activated $\mathrm{CD} 4^{+} \mathrm{T}$ cell subsets suggests modulation of $\mathrm{CD}^{+} \mathrm{T}$ cell activation by MФs.

Conclusion: SF MD subsets are associated with knee OA PROMs and display an activated phenotype, which may lead to modulation of $\mathrm{CD}^{+} \mathrm{T}$ cell activation. Knee OA SF MФ subsets could serve as knee OA function biomarkers and as targets of novel therapeutics.

Keywords: Osteoarthritis, Synovial fluid, Monocytes/macrophages, PROMs, Leukocytes

\footnotetext{
* Correspondence: sviswana@uhnresearch.ca

${ }^{1}$ Arthritis Program, University Health Network, Toronto, ON, Canada

${ }^{2}$ Krembil Research Institute, University Health Network, Toronto, ON, Canada

Full list of author information is available at the end of the article
}

(c) The Author(s). 2019 Open Access This article is distributed under the terms of the Creative Commons Attribution 4.0 International License (http://creativecommons.org/licenses/by/4.0/), which permits unrestricted use, distribution, and reproduction in any medium, provided you give appropriate credit to the original author(s) and the source, provide a link to the Creative Commons license, and indicate if changes were made. The Creative Commons Public Domain Dedication waiver (http://creativecommons.org/publicdomain/zero/1.0/) applies to the data made available in this article, unless otherwise stated. 


\section{Background}

Osteoarthritis (OA) is increasingly considered a chronic, low-grade inflammatory disease with involvement of synovial inflammation (synovitis) [1]. Synovitis promotes an inflammatory environment associated with cartilage degradation [2]. In fact, synovitis predicts OA progression and is associated with pain and cartilage degradation $[3,4]$.

The main immune cells (leukocytes) present in the OA synovium are monocytes/macrophages (defined as a heterogeneous mixture of monocytes and macrophages, MФs) typically found along the synovial lining layer [5]. MФs from OA synovium produce inflammatory and degradative mediators [6] and ablation of synovial-resident $\mathrm{M} \Phi$ s reduces OA severity in a murine model [7]. Thus, there is evidence that MФs contribute to the OA pathogenesis.

Synovitis is often associated with knee fluid effusion [2], suggesting that changes in the synovial fluid (SF) environment happen along with changes in the synovium. Thus, while leukocyte numbers in OA SFs are low $\left(<2000 / \mathrm{mm}^{3}\right)$, these cells may provide information on the inflammatory state of the joint or the patient's state. Types of leukocytes in knee OA (KOA) SF [811] have been quantified: with $M \Phi s$ in synovial fluid leukocytes (SFLs) showing considerable heterogeneity between studies, with reported frequencies of SFLs of $3-34 \%$ [8-11]. Some of these studies have attempted to characterize subpopulations of SF MФs $[10,11]$ but the association between SF MФs and the clinical outcomes has not been studied.

The importance of $\mathrm{M} \Phi$-specific factors (i.e. $\mathrm{sCD} 14$ and sCD163) [12] and MФ chemoattractants (CCL2 and CX3CL1) [13-16] have also been investigated in OA SF, and they have been identified as being associated with clinical outcomes [12, 14, 16]. However, the association between these $M \Phi$-specific factors and chemoattractants and specific $M \Phi$ subpopulations has not been studied. This is an important area of study as there is growing interest in understanding the effects of $M \Phi$ subpopulations on OA pathology.

In circulation, $\mathrm{M} \Phi$ s are classified into three main subtypes based on cell surface antigens: $\mathrm{CD} 14^{+} \mathrm{CD} 16^{\text {neg }}$ (classical), $\mathrm{CD} 14^{+} \mathrm{CD} 16^{+}$(intermediate) and $\mathrm{CD} 14^{\text {low }} \mathrm{CD} 16^{+}$ (non-classical). $\mathrm{CD} 14^{+} \mathrm{CD} 16^{\text {neg }} \mathrm{M \Phi s}$ are the most abundant ( 85\%), followed by CD16 $6^{+} \mathrm{CD} 14^{\text {low }}(\sim 10 \%)$ and the double-positive $\mathrm{CD} 14^{+} \mathrm{CD} 16^{+}$subset $(\sim 5 \%)$ [17]. The double-positive $\mathrm{CD} 14^{+} \mathrm{CD} 16^{+}-\mathrm{M} \Phi$ subset frequency increases in systemic inflammation and inflammatory diseases such as rheumatoid arthritis (RA), Crohn's disease, Eales' disease and asthma [17-19]. Double-positive $\mathrm{CD} 14^{+} \mathrm{CD} 16^{+}$-MФs are considered pro-inflammatory, among the three $М \Phi$ subsets, as they preferentially induce $\mathrm{T}$ cell activation, have superior production of reactive oxygen species and express the highest levels of human leukocyte antigen-antigen D related (HLA-DR), involved in antigen presentation to $\mathrm{CD} 4^{+} \mathrm{T}$ cells $[17,18,20]$.

To date no manuscript has been published on MФ subsets in OA SF, as defined by CD14 and CD16 markers; however, studies in RA and juvenile idiopathic arthritis SFs show that the double-positive $\mathrm{CD} 14^{+} \mathrm{CD} 16^{+}-\mathrm{M} \Phi$ subset is increased in SF MФs when compared to circulating MФs [21-23]. Similar to circulating MФs, RA SF double-positive $\mathrm{CD} 14^{+} \mathrm{CD} 16^{+}-\mathrm{M} \Phi$ express the highest levels of HLA-DR [22]. The specific function of SF MФ subsets has not been analyzed in any disease; however, the overall RA SF MФs promote the secretion of inflammatory factors, interleukin- 17 and interferon- $\gamma$, by $\mathrm{T}$ helper cells [21, 24]. This suggests a possible association between higher levels of SF double-positive $\mathrm{CD} 14^{+} \mathrm{CD} 16^{+}-\mathrm{M} \Phi \mathrm{s}$ and joint inflammation, at least in RA.

In this cohort study, we investigated the SFL populations and tested whether the double-positive $\mathrm{CD} 14^{+} \mathrm{CD} 16^{+}-\mathrm{M} \Phi$ subset in SFLs from patients with knee KOA is linked to patient-reported outcome measures (PROMs) and soluble SF M $\Phi$ factors. In addition, we determined whether SF MФs are associated with SF $\mathrm{CD} 4^{+} \mathrm{T}$ cell activation.

\section{Patients and methods}

\section{Patients with KOA}

Patients diagnosed with KOA $(\mathrm{n}=86)$ donated their SF at the time of clinical intervention (intra-articular injection, arthroscopy or arthroplasty). Blood from $53 \mathrm{pa}-$ tients with $\mathrm{KOA}$ was also acquired, 40 which were also SF donors (Fig. 1a). Patients provided written informed consent to participate in this study, approved by the University Health Network Research Ethics Board (Protocol ID 14-7483-AE). All enrolled patients met the American College of Rheumatology criteria for symptomatic KOA [25]. Exclusion criteria included history of inflammatory arthritis, intra-articular corticosteroid injection (within 3 weeks of surgery) and blood dyscrasias.

Radiographs from 78 of the 86 patients were available for Kellgren-Lawrence (KL) grading. Radiographs were scored by an experienced surgeon for KL grade. Early-stage KOA was defined as KL grades I/II and late-stage as grades III/IV.

\section{Assessment of PROMs}

Functional and symptomatic assessment was evaluated using PROMs. Briefly, Knee Injury and Osteoarthritis Injury Score (KOOS) questionnaires [26] were filled out prior to clinical intervention (up to 3 months before the intervention, $N=83$; 3 patients who donated SF did not fill out questionnaires). Symptoms, pain, activity of daily living (ADL), sports and quality of life (QOL) KOOS subscales, and Western Ontario and McMaster Universities Osteoarthritis Index (WOMAC) pain, 


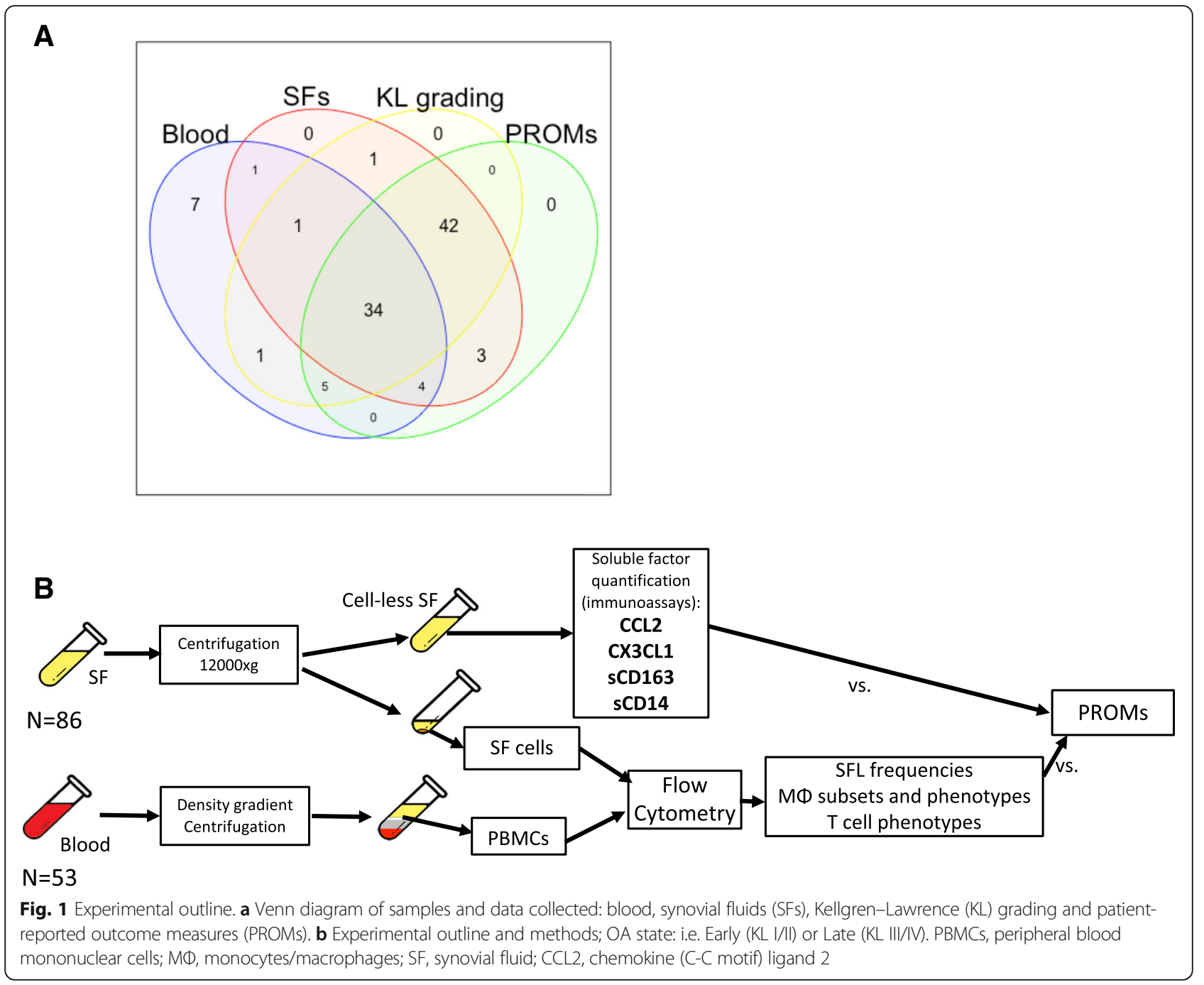

stiffness and function were determined from the questionnaire responses [26]. Mean KOOS and WOMAC were determined by averaging all KOOS and WOMAC subscales, respectively. All KOOS and WOMAC scores are presented in a scale from 0 to 100 , where 0 is the worst outcome.

\section{SF and peripheral blood mononuclear cell (PBMC) isolation}

SFs and blood were acquired at the time of the intervention, stored at $4{ }^{\circ} \mathrm{C}$ and processed within $24 \mathrm{~h}$ of acquisition (Fig. 1b). Further details are in Additional file 1: Supplementary Methods.

\section{Measurement of cell populations in SFs and PBMCs}

MФs and their subpopulations were identified based on Abeles et al., 2012 [27] (Additional file 1: Figure S1), using FlowJo v10. Neutrophils were identified as $\mathrm{CD} 6^{+}$HLA-DR $^{\text {neg }}$ with high granularity while natural killer (NK) cells are those with low granularity. МФs were $\mathrm{CD} 14^{+}$and/or $\mathrm{CD} 16^{+}$while expressing HLA-DR at different levels. Additional details on cell labelling are presented in Additional file 1: Supplementary Methods.

\section{Measurements of chemotactic factors and soluble receptors}

SF supernatants were digested with hyaluronidase, to reduce viscosity, using a 1:1 SF: hyaluronidase solution in RPMI at $500 \mathrm{U} / \mathrm{ml}$ for $1 \mathrm{~h}$ at room temperature with circular shaking $(600 \mathrm{rpm})$ (Fig. 1b). The chemokine (C-C motif) ligand 2 and chemokine (C-X3-C motif) ligand 1 (CX3CL1) were detected using ProcartaPlex simplex assay as per manufacturer's recommendations (ThermoFisher). The intra-assay and inter-assay CV was $7.5 \%$ and $14.2 \%$, respectively, for CCL2, and the intra-assay and inter-assay CV for CX3CL1 was $10 \%$. A dilution of 1:10 was used (with kit=specific buffer) to reduce interference of SF matrix on the assay. SCD14 (x 1000 dilution) and sCD163 (x 400 dilution) were 
detected using ELISA kits and diluted with kit-specific solutions (ThermoFisher); the inter-assay and the intra-assay $\mathrm{CV}$ is $<12 \%$ and $<10 \%$, respectively.

\section{Statistical analysis}

Descriptive statistics were obtained (Additional file 1: Tables S1 and S2). The Wilcoxon sign-rank (paired) test was used to compare blood and SF samples and to compare between populations within the same sample. Bootstrapping was used to perform two-sided tests for estimation of Pearson correlation coefficients and linear modeling on samples to analyze the empirical distribution [28] and thus overcome the need to assume normality in either the samples or residuals; however the $t$ values were confirmed as normal by histogram and normal quantile plot after bootstrap; 10,000 bootstrapping samples were taken and $P$ values calculated by evaluating the hypothesis of $\mathrm{P}(r=0 \mid \mathrm{H} 0)$ using bootstrapped estimates [29] and the adjusted $P$ value was acquired by adjusting for multiple comparisons (for each set of comparisons) as per the method of Li and Ji [30]: briefly, the correlation matrix of the parameters evaluated for each set of comparisons (e.g. cell population frequencies, PROMs) was calculated, followed by estimating the effective number of comparisons (Meff) from the eigen values $(\lambda)$ of the correlation matrix as per the equation $\mathrm{Li}$ and Ji [30] proposed; the adjusted $p$ value was then calculated as per the equation:

\section{1-(1- $p$ value $)^{\text {Meff }}$}

Confidence intervals $(\mathrm{CI})$ were calculated using the adjusted bootstrap percentile. For linear models, the effect estimates $(\beta)$, which indicate the slope for the given variable, is presented along CIs. Our linear models are adequately powered for a multiple $\mathrm{R}$ square of 0.133 (using four predictors) or 0.154 (using five predictors), alpha $=0.05$ and power $=0.8$.

\section{Results}

The median age and body mass index (BMI) of our cohort ( $n=83$, used for linear modeling) was 62 years and 29.75 $\mathrm{kg} / \mathrm{m}^{2}$, respectively: $60 \%$ of patients were female; $49 \%$ underwent arthroplasty. Within the patients graded by KL $(N=76), 31.6 \%$ were in early-stage KOA (KLI/II). Median values for KOOS and WOMAC subscales were 44.4-50 for WOMAC and KOOS pain, KOOS ADL, WOMAC function and WOMAC stiffness. The medians for KOOS QOL and KOOS sports were 18.8 and 15, respectively (Additional file 1: Table S1). Use of pain medications is tabulated in Additional file 1: Table S2; and no effects on $M \Phi$ subset frequencies in total SF MФs was seen from use of pain medications (Additional file 1: Figure S2).

\section{Leukocytes in KOA SF}

The main leukocyte populations in KOA SFLs were MФs $($ median $=36.5 \%)$, followed by $\mathrm{T}$ cells $($ median $=31.1 \%$; with $p<0.001$ for T cells vs. MФs, Fig. 2a). Neutrophils were not abundant in KOA SFs, with a median SFL frequency of $2.95 \%$; only $12 \%(N=75)$ of patients had $>20 \%$ neutrophils in their SFLs.

Since most KOA SFLs (>97.0\%) are mononuclear, PBMCs $(N=40)$ were used as a control to benchmark SFLs. Relative to circulation, MФs were enriched in KOA SFLs while $\mathrm{T}$ and NK cells were diminished (Fig. 2a).

Further analysis of $\mathrm{M} \Phi$ subsets using CD14 and CD16 markers, typically used for classifying blood monocytes [17] (Additional file 1: Figure S1), showed that KOA SF MФs are selectively enriched for the double-positive $\mathrm{CD} 4^{+} \mathrm{CD} 16^{+}$subset $(39.4 \%$ vs. $6.3 \%$ in circulation); both
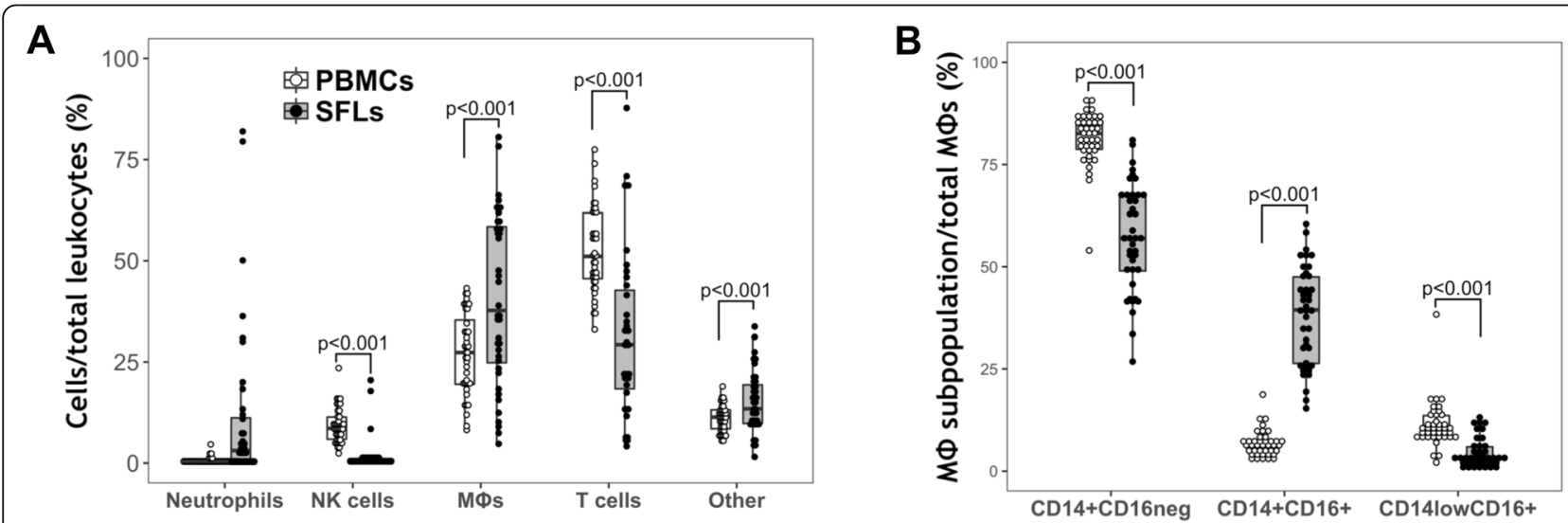

Fig. 2 Knee osteoarthritis (KOA) synovial fluid (SF) monocytes/macrophages (MDs) are the most abundant SF leukocytes (SFLs) and have different prevalence to those in the circulation. a Frequency of SFL and peripheral blood mononuclear cell (PBMC) $(N=40)$ populations. No statistical analysis is shown for neutrophils. b MФ subset ratios, in PBMC and SF MФs $(N=40)$. a and $\mathbf{b}$ Wilcoxon signed test (paired) was used. $P$ values shown are corrected for multiple comparisons. Box plots indicate median with interquartile range for box and Tukey-style whiskers. Lines between boxplots indicate significant differences, with adjusted $p$ value shown on top. White dots with white box represent data from PBMCs; black dots with gray box represent data from SFLs. NK, natural killer 
$\mathrm{CD} 4^{+} \mathrm{CD} 16^{\text {neg }}(57.00 \%)$ and $\mathrm{CD} 14^{\text {low }} \mathrm{CD} 16^{+}(3.34 \%)$ subsets were reduced in frequency compared to circulating MФs in patients with KOA $(82.5 \%$ and $10.7 \%$ for $\mathrm{CD} 14^{+} \mathrm{CD} 16^{\text {neg }}$ and $\mathrm{CD} 14^{\text {low }} \mathrm{CD} 16^{+}$, respectively) (Fig. 2b). Interestingly, some MФs in OA SF expressed the mature macrophage marker 25F9 [31]. This marker was not expressed on circulating MФs, indicating the lack of mature macrophages. There was higher frequency of $25 \mathrm{~F} 9$ expression on SF CD14 ${ }^{\text {low }} \mathrm{CD} 16^{+}-\mathrm{M} \Phi \mathrm{s}(30.9 \%)$ than on SF double-positive $\mathrm{CD} 14^{+} \mathrm{CD} 16^{+}$-MФs (17.3\%), and only a few 25F9+ MФs in the $\mathrm{CD} 14^{+} \mathrm{CD} 16^{\text {neg }}$ subset $(5.43 \%)$ (Additional file 1: Figure S3C).

These data indicate that $\mathrm{M} \Phi \mathrm{s}$ are the most abundant SFLs and are enriched for the putative pro-inflammatory double-positive $\mathrm{CD} 14^{+} \mathrm{CD} 16^{+}$-MФ subset.

\section{$M \Phi$ subset ratios correlate with PROMs}

Linear modeling, adjusted for sex, BMI and age, known confounders of KOA severity [32-34], showed that both $\mathrm{CD} 14^{+} \mathrm{CD} 16^{\text {neg }}{ }_{-\mathrm{M}} \mathrm{Ms} /$ total SF MФs and double-positive
$\mathrm{CD} 14^{+} \mathrm{CD} 16^{+}$-MФs/total SF MФs are significant predictors of mean KOOS and WOMAC scores (Table 1), with a $1 \%$ increase in either of these $M \Phi$ ratios leading to a 0.38-0.47-point change in mean KOOS or WOMAC scores. These two MФ subsets were closely correlated, as they comprised $96.4 \%$ of all SF MФs; an increase in one subset would typically come at the expense of the other subset. With this in mind, CD14 ${ }^{+} \mathrm{CD} 16^{\text {neg }}-\mathrm{M} \Phi$ s/total SF MФs was a significant predictor of all subscales except KOOS-symptoms, WOMAC-pain and KOOS-pain subscales. The double-positive $\mathrm{CD} 14^{+} \mathrm{CD} 16^{+}-\mathrm{M} \Phi \mathrm{s} /$ total SF MФs displayed more specificity for predicting KOOS-QOL and WOMAC-stiffness than $\mathrm{CD} 14^{+} \mathrm{CD} 16^{\text {neg }}-\mathrm{M} \Phi$ s/total SF MФs (Table 1). A $1 \%$ change in the double-positive $\mathrm{CD}_{14}{ }^{+} \mathrm{CD} 16^{+}-\mathrm{M} \Phi$ s/total SF $\mathrm{M \Phi s}$ ratio affects KOOS-QOL and WOMAC-stiffness scores by 0.57 and 0.58 points, respectively, vs. 0.45 for both subscales from a $1 \%$ change in the $\mathrm{CD} 14^{+} \mathrm{CD} 16^{\text {neg }}{ }_{-\mathrm{M}} \Phi \mathrm{s} /$ total SF MФs ratio.

Interestingly, the third subset, $\mathrm{CD} 14^{\text {low }} \mathrm{CD} 16^{+}-\mathrm{M} \Phi \mathrm{s} /$ total SF MФs was not associated with mean KOOS or

Table 1 Effect size of ratio of MФ subsets in total SF MDs with patient reported outcome measures (PROMs)

\begin{tabular}{|c|c|c|c|}
\hline & $\mathrm{CD} 14^{+} \mathrm{CD} 16^{\text {neg }}-\mathrm{M} \Phi \mathrm{s} /$ total SF MФs (\%) & $\mathrm{CD} 4^{+} \mathrm{CD} 16^{+}$-MФs/total SF MФs (\%) & CD14 ${ }^{\text {low }}$ CD $16^{+}-M \Phi s /$ total SF MФs (\%) \\
\hline & $\beta(95 \% \mathrm{Cl})$, adjusted $p$ & $\beta(95 \% \mathrm{Cl})$, adjusted $p$ & $\beta(95 \% \mathrm{Cl})$, adjusted $p$ \\
\hline \multicolumn{4}{|l|}{ Overall } \\
\hline mean KOOS & $0.379(0.179,0.610), p=0.006$ & $-0.376(-0.642,-0.132), p=0.025$ & $-0.761(-1.601,-0.030), p=0.115$ \\
\hline mean $\mathrm{KOOS}^{\mathrm{a}}$ & $0.380(0.193,0.613), p=0.003$ & $-0.415(-0.682,-0.178), p=0.005$ & $-0.545(-1.191,0.048), p=0.247$ \\
\hline mean WOMAC & $0.431(0.191,0.710), p=0.010$ & $-0.459(-0.769,-0.170), p=0.018$ & $-0.734(-1.738,0.059), p=0.224$ \\
\hline mean WOMAC ${ }^{a}$ & $0.380(0.193,0.613), p=0.003$ & $-0.473(-0.800,-0.159), p=0.023$ & $-0.500(-1.335,0.138), p=0.463$ \\
\hline \multicolumn{4}{|l|}{ KOOS } \\
\hline SYMPTOMS & $0.187(-0.055,0.453), p=0.459$ & $-0.177(-0.491,0.114), p=0.667$ & $-0.372(-1.227,0.479), p=0.869$ \\
\hline SYMPTOMS ${ }^{a}$ & $0.188(-0.040,0.471), p=0.437$ & $-0.228(-0.539,0.045), p=0.389$ & $-0.153(-0.904,0.573), p=0.988$ \\
\hline PAIN & $0.299(0.066,0.556), p=0.069$ & $-0.295(-0.594,0.013), p=0.215$ & $-0.633(-1.526,0.069), p=0.271$ \\
\hline PAIN $^{a}$ & $0.288(0.023,0.579), p=0.156$ & $-0.308(-0.642,0.016), p=0.244$ & $-0.450(-1.195,0.202), p=0.554$ \\
\hline $\mathrm{ADL}$ & $0.477(0.202,0.796), p=0.013$ & $-0.458(-0.816,-0.117), p=0.054$ & $-1.094(-2.214,-0.169), p=0.052$ \\
\hline $\mathrm{ADL}^{\mathrm{a}}$ & $0.482(0.201,0.797), p=0.010$ & $-0.492(-0.845,-0.137), p=0.038$ & $-0.861(-1.788,-0.127), p=0.092$ \\
\hline QOL & $0.431(0.170,0.702), p=0.010$ & $-0.497(-0.792,-0.215), p=0.008$ & $-0.463(-1.508,0.432), p=0.813$ \\
\hline $\mathrm{QOL}^{\mathrm{a}}$ & $0.453(0.253,0.667), p=0.002$ & $-0.571(-0.826,-0.357), q<0.001$ & $-0.231(-0.954,0.459), p=0.932$ \\
\hline SPORTS & $0.502(0.223,0.869), p=0.009$ & $-0.454(-0.901,-0.101), p=0.104$ & $-1.244(-2.164,-0.512), p=0.005$ \\
\hline SPORTS ${ }^{a}$ & $0.491(0.213,0.865), p=0.014$ & $-0.475(-0.925,-0.122), p=0.071$ & $-1.033(-1.839,-0.347), p=0.031$ \\
\hline \multicolumn{4}{|l|}{ WOMAC } \\
\hline PAIN & $0.322(0.084,0.608), p=0.059$ & $-0.327(-0.659,-0.035), p=0.137$ & $-0.655(-1.616,0.116), p=0.330$ \\
\hline PAIN $^{a}$ & $0.312(0.040,0.616), p=0.122$ & $-0.347(-0.711,-0.016), p=0.167$ & $-0.378(-1.203,0.318), p=0.753$ \\
\hline FUNCTION & $0.477(0.202,0.795), p=0.013$ & $-0.458(-0.816,-0.117), p=0.054$ & $-1.093(-2.213,-0.168), p=0.053$ \\
\hline FUNCTION & $0.482(0.201,0.797), p=0.010$ & $-0.492(-0.845,-0.137), p=0.037$ & $-0.860(-1.788,-0.127), p=0.092$ \\
\hline STIFFNESS & $0.479(0.194,0.788), p=0.010$ & $-0.592(-0.922,-0.274), p=0.007$ & $-0.454(-1.517,0.413), p=0.815$ \\
\hline STIFFNESS ${ }^{a}$ & $0.453(0.159,0.777), p=0.019$ & $-0.580(-0.924,-0.247), p=0.010$ & $-0.262(-1.217,0.450), p=0.951$ \\
\hline
\end{tabular}

All correlations adjusted for sex, body mass index and age; $N=83$. Values in boldface show results with significant effect estimates ( $\beta$ )

$M \Phi$ monocytes/macrophages, SF synovial fluid, SFLs synovial fluid leukocytes, KOOS Knee Injury and Osteoarthritis Injury Score, WOMAC Western Ontario and McMaster Universities Osteoarthritis Index, $A D L$ activities of daily living, QOL quality of life

${ }^{a}$ Additionally adjusted for osteoarthritis state (i.e. early, Kellgren-Lawrence (KL) grade I/II, $n=24$ or late, KL grade III/IV, $n=52$ ); $N=76$ 
WOMAC but was a good predictor of the KOOSsports subscale. These data reveal that МФ subset ratios can indicate different aspects of functional outcomes in patients with KOA.

\section{MФ-shed receptors are not associated with PROMs}

Levels of sCD14 and sCD163, shed from MФs, have previously been associated with structural changes in $\mathrm{KOA}$ and in the case of sCD14, with KOA symptoms [12]. We investigated whether these shed receptors correlate with specific $\mathrm{M} \Phi$ subsets; levels of $\mathrm{sCD} 14$ and sCD163 in KOA SF correlated with each other $(r=$ $0.630 p<0.001$ ), as previously shown [12] (Additional file 1: Figure S5). In our hands, however, neither sCD163 nor sCD14 were predictors of any PROMs upon adjustment for sex, age and BMI, or additional adjustment for OA stage (Additional file 1: Table S5). There was significant correlation between levels of SF sCD14 and double-positive $\mathrm{CD} 14^{+} \mathrm{CD} 16^{+}$-MФs/SFLs, reflective of correlation between $\mathrm{SCD} 14$ and the overall frequency of $\mathrm{M} \Phi \mathrm{s}$ in SFLs (Table 2, Additional file 1: Figure S7).

These data indicate that there is no association between SF MФ subset ratios (in total MФs) and the levels of shed receptors, however the correlation between sCD14 and the overall frequency of MФs and the double-positive $\mathrm{CD} 14^{+} \mathrm{CD} 16^{+}$subset in SFLs reveals associations between $M \Phi$ frequencies to previously investigated biomarkers of $\mathrm{OA}$ severity.

\section{SF KOA CD $14^{+} \mathrm{CD} 16^{+} \mathrm{M} \Phi$ subset is associated with SF CCL2 levels}

We investigated whether the level of CCL2, an important $M \Phi$ chemoattractant [17] and agonist of CCR2, is associated with the ratios of MФ subsets from OA SF. CCL2 was present at $343.2 \mathrm{pg} / \mathrm{ml}$ (median) in KOA SF and its levels correlated with the ratio of double-positive $\mathrm{CD} 14^{+} \mathrm{CD} 16^{+}$-MФs/total SF MФs (adjusted $p=0.004$, Fig. 3). There was no significant correlation between CCL2 and the other MФ subsets (Additional file 1: Table S6) and CCL2 was not associated with PROMs either
(Additional file 1: Table S7). Interestingly, SF doublepositive $\mathrm{CD} 14^{+} \mathrm{CD} 16^{+}$-MФs had the highest frequency of $\mathrm{CCR}^{+}$cells $(89.1 \%$, Additional file 1: Figure S3A) and expressed the highest levels of CCR2 per cell among SF $\mathrm{M} \Phi$; $\quad$ conversely circulating $\mathrm{CD} 14^{+} \mathrm{CD} 16^{\text {neg }}-\mathrm{M} \Phi \mathrm{s}$ expressed the highest levels of CCR2 per cell (Additional file 1: Figure S3B).

An alternative modulation of the SF MФ ratio could be through CX3CL1, a chemokine that signals to both double-positive $\mathrm{CD} 14^{+} \mathrm{CD} 16^{+}$and $\mathrm{CD} 14^{\text {low }} \mathrm{CD} 16^{+} \mathrm{M} \Phi$ subsets [20]. However, we were unable to detect this chemokine in KOA SFs (lowest detection limit $11.7 \mathrm{pg} / \mathrm{ml}$ ).

\section{KOA SF MФs are in an activated state}

HLA-DR is one of the major histocompatibility complexes and is used by pro-inflammatory MФs to present antigens to $\mathrm{CD} 4^{+} \mathrm{T}$ cells, leading to their activation [35]. MФs in KOA SFs have higher expression of HLA-DR compared to those in circulation $(N=40$, Fig. 4a), indicating they are in an activated state [36]. HLA-DR expression (on a per cell basis) is the highest on SF double-positive $\mathrm{CD} 14^{+} \mathrm{CD} 16^{+}$-MФs (Fig. 4a), supportive of a pro-inflammatory function in this subset.

Further evidence of pro-inflammatory $\mathrm{M} \Phi$ activation was investigated indirectly by determining the activation of SF CD4 $4^{+} \mathrm{T}$ cells and their associations with $\mathrm{M} \Phi$ subsets. Thus, using markers of early (CD69), intermediate (CD25) and late $\mathrm{T}$ cell activation (HLA-DR), we determined that $71.70 \%$ of $\mathrm{KOA} \mathrm{SF} \mathrm{CD}^{+}{ }^{+} \mathrm{T}$ cells are $\mathrm{CD} 9^{+}, 19.94 \%$ are $\mathrm{CD} 25^{+}$and $36.35 \%$ are HLA-DR ${ }^{+}$, indicating a spectrum of activated $\mathrm{T}$ cells in KOA SFs (Fig. 4b). We also found negative correlation between the ratio of double-positive $\mathrm{CD} 14^{+} \mathrm{CD} 16^{+}-\mathrm{M} \Phi /$ total SF $\mathrm{M} \Phi$ s vs. $\mathrm{CD} 9^{+} / \mathrm{CD} 4^{+} \mathrm{T}$ cells (Fig. 4c), and the ratio of $\mathrm{CD} 14^{+} \mathrm{CD} 16^{\text {neg }}-\mathrm{M} \Phi /$ total SF MФs vs. HLA-DR ${ }^{+} / \mathrm{CD} 4^{+}$ $T$ cells (Fig. 4d). Taken together, KOA SF MФ subsets were associated with the degree of $\mathrm{SF} \mathrm{CD}^{+} \mathrm{T}$ cell activation, indicating further evidence of an activated MФ state.

Table 2 Correlation between MФ subsets and SF sCD163 and sCD14

\begin{tabular}{|c|c|c|c|c|}
\hline & \multicolumn{2}{|l|}{ sCD163 (ng/ml) } & \multicolumn{2}{|l|}{ sCD14 (ug/ml) } \\
\hline & Pearson's $r, \mathrm{Cl}$ & $\overline{\text { Adjusted } p}$ & Pearson's $r, \mathrm{Cl}$ & $\overline{\text { Adjusted } p}$ \\
\hline $\mathrm{CD}_{14}{ }^{+} \mathrm{CD} 16^{+}-\mathrm{M} \Phi \mathrm{s} /$ total SF MФs & $-0.007(-0.263,0.232)$ & 1.000 & $-0.044(-0.306,0.226)$ & 0.996 \\
\hline $\mathrm{CD} 14^{+} \mathrm{CD} 16^{\text {neg }}$-MФs /total SF MФs & $0.009(-0.231,0.250)$ & 1.000 & $0.073(-0.185,0.318)$ & 0.967 \\
\hline $\mathrm{CD} 14^{\text {low }} \mathrm{CD} 16^{+}$-MФs /total SF MФs & $-0.008(-0.186,0.270)$ & 1.000 & $-0.110(-0.300,0.091)$ & 0.708 \\
\hline Total MФs/total SFLs & $-0.147(-0.334,0.074)$ & 0.695 & $-0.304(-0.491,-0.073)$ & 0.028 \\
\hline $\mathrm{CD} 14^{+} \mathrm{CD} 16^{+}-\mathrm{M} \Phi$ s/total SFLs & $-0.131(-0.329,0.097)$ & 0.654 & $-0.294(-0.482,-0.054)$ & 0.043 \\
\hline $\mathrm{CD} 14^{+} \mathrm{CD} 16^{\text {neg }}-\mathrm{M} \Phi$ s/total SFLs & $-0.134(-0.328,0.090)$ & 0.614 & $-0.225(-0.428,0.000)$ & 0.165 \\
\hline $\mathrm{CD} 14^{\text {low }} \mathrm{CD} 16^{+}-\mathrm{M} \Phi$ s/total SFLs & $-0.053(-0.218,0.226)$ & 0.969 & $-0.223(-0.390,-0.018)$ & 0.111 \\
\hline
\end{tabular}

$N=81$. Values in boldface show results with significant effect estimates $(\beta)$

$M \Phi$ monocytes/macrophages, SF synovial fluid, SFL, synovial fluid leukocytes 


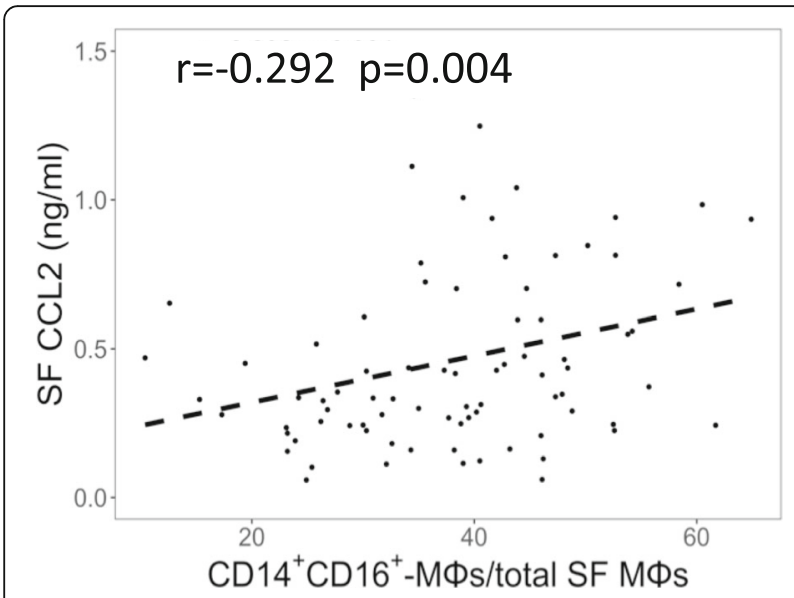

Fig. 3 Pro-inflammatory monocytes/macrophages $(M \Phi)$ subset correlate with synovial fluid (SF) chemokine (C-C motif) ligand 2 (CCL2) levels. Positive correlation between SF CCL2 levels and CD14 ${ }^{+}$ CD $16^{+}-\mathrm{MDs}$ (to total SF MDs) ratio, $N=81$. Pearson $r$ and adjusted $p$ value. Dashed line indicates regression resulting from general linear modeling approximation

\section{Discussion}

To our knowledge, this is the first study to investigate the ratios of KOA SF M $\Phi$ subsets and their association with patient-reported outcomes and SF soluble factors. We provide evidence that (1) SF MФs in KOA have a high prevalence of putative pro-inflammatory subtypes; (2) SF MФ subsets are associated with PROMs; and (3) SF MФs are activated and associated with SF $\mathrm{CD}^{+}{ }^{+} \mathrm{T}$ cell activation, potentially promoting a more adaptive immune response.

Our data on SFL populations are comparable to other reports $[10,11]$. However, they also differ from frequencies reported by Penatti et al. [9] or Jónasdóttir et al. [8]; the differences observed in those two reports can be attributed to the differences in SFL isolation from the SF: both reported using centrifugation forces lower than $2000 \times$ g, which based on our experience, with the high viscosity of most KOA SF samples, does not allow for full cell pelleting, with many cells remaining in suspension.

Interestingly, our profile of SF MФs is similar to the SF MФs profile in RA [21, 22]; although the RA SFLs are considerably more abundant than OA's, consistent with marked inflammation of the synovium in RA [21]. Similar to our reports, the double-positive $\mathrm{CD} 14^{+} \mathrm{CD} 16^{+}-\mathrm{M} \Phi$ subset is also increased in RA SF MФs when compared to circulating MФs [21].

We showed, for the first time, that SF MФ subsets are partially explanatory of KOOS and WOMAC scores. Both $\mathrm{CD} 14^{+} \mathrm{CD} 16^{\text {neg }}$ and $\mathrm{CD} 14^{+} \mathrm{CD} 16^{+}-\mathrm{M} \Phi$ subsets were good predictors of mean KOOS and WOMAC scores; we think that since an increase in one subset (i.e. double-positive $\mathrm{CD} 14^{+} \mathrm{CD} 16^{+}$-MФs) typically comes at the expense of the other subset (i.e. CD14

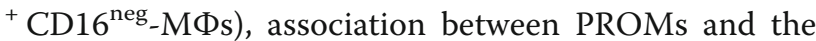
$\mathrm{CD} 14^{+} \mathrm{CD} 16^{\text {neg }}-\mathrm{M} \Phi$ s/total SF MФs ratio is a result of an active increase in the double-positive $\mathrm{CD} 14^{+} \mathrm{CD} 16^{+}$-MФs. We thus hypothesize that double-positive $\mathrm{CD} 14^{+} \mathrm{CD} 16^{+}$-MФs may be the actual effectors that lead to changes in patients' function and quality of life as they had higher effect estimates $(\beta)$ for WOMAC stiffness and KOOS quality of life. Importantly, none of the associations between PROMs and $M \Phi$ subset ratios were dependent on radiographic OA stage. This supports previous results showing correlation between synovitis scores and pain, but not radiographic OA grading [3]. However, we did observe higher effect estimates in subcohorts with early stage vs. late stage $\mathrm{KOA}$.

Our data on sCD14 differed from parts of the data reported by Daghestani et al. [12]. We show that sCD14 is correlated with some KOA PROMs (KOOS and WOMAC vs. First National Health and Nutrition Examination Survey criterion [12]), but only when not adjusted for confounders. The lack of correlation with adjustments may be due to differences in cohorts as previously noted [12] and the different criteria used to evaluate PROMs. Similarly, we did not find any significant association between CCL2 levels and PROMs and we failed to detect any CX3CL1 in the SFs from our cohort. This differs from the report from Li and Jian [16], which showed a significant association between SF CCL2 levels and WOMAC subscales; interestingly their median SF CCL2 of $44.8 \mathrm{ng} / \mathrm{ml}$ was at least two orders of magnitude greater, indicating that cohorts are significantly different. Similarly, the measurable levels of CX3CL1 by Huo et al. [14] in OA SF and its association with PROMs may be related to the cohort chosen.

We speculate that the measurable levels of the chemokine CCL2 present in KOA SFs indicate a mechanism of SF MФ recruitment from circulation, which is corroborated by expression of CCR2 on most SF MФs. Expression of CCR2 (exclusive to monocytes rather than macrophages [31]) suggests presence of recruited circulating monocytes into KOA SFs rather than presence of resident macrophages shed from the synovial lining. Murine studies also show that inhibition of CCL2/CCR2 signaling leads to decreased synovial MФs [37-39], indicating less МФ recruitment, and concomitant reduction in pain $[37,38]$ and cartilage degradation [39]. In addition, the expression of the mature macrophage marker, 25F9 [31] at higher proportions on SF $\mathrm{CD} 14^{\text {low }}{ }^{2} \mathrm{CD} 16^{+}-\mathrm{M} \Phi \mathrm{s}(30.9 \%)$ than on SF double-positive $\mathrm{CD} 14^{+} \mathrm{CD} 16^{+}$-MФs $(17.3 \%)$ suggests that SF MФs are likely recruited from circulation as $\mathrm{CD} 14^{+} \mathrm{CD} 16^{\mathrm{neg}}$-monocytes, subsequently maturing into double-positive $\mathrm{CD} 14^{+} \mathrm{CD} 16^{+}-\mathrm{M} \Phi \mathrm{s}$, and finally into $\mathrm{CD} 14^{\text {low }} \mathrm{CD} 16^{+}$-MФs [40], based on joint environmental cues. 


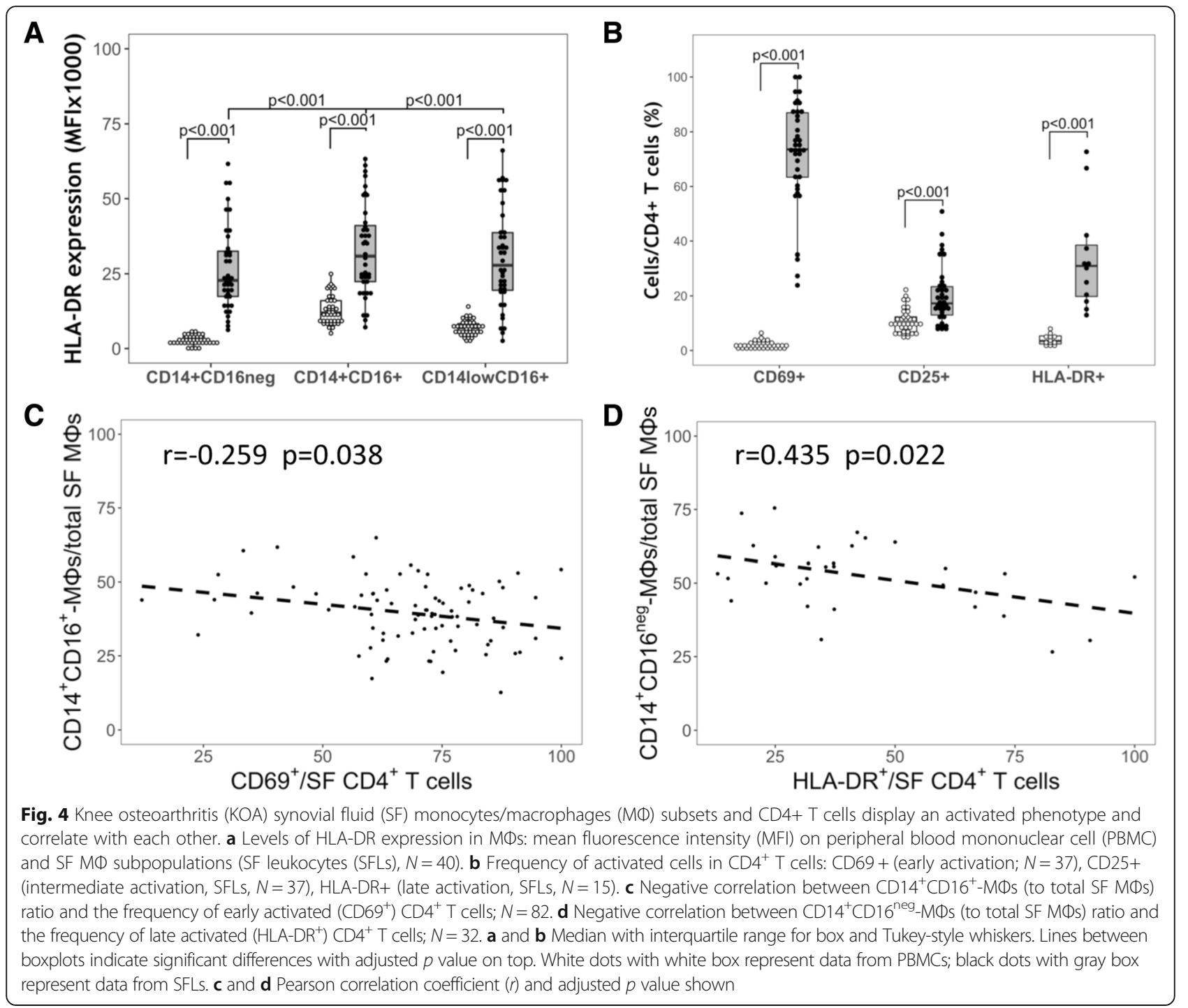

The activation state of KOA SF MФs has previously been reported for SF MФs in RA where SF MФs regulate $\mathrm{CD}^{+} \mathrm{T}$ cell responses [21]. Our data show that KOA SF MФ subsets are associated with the levels of early and late activation in $\mathrm{CD} 4^{+} \mathrm{T}$ cells, suggesting that KOA SF MФ subsets may functionally serve as possible modulators of SF CD4 ${ }^{+} \mathrm{T}$ cell activation. Specifically, we hypothesize that SF double-positive $\mathrm{CD} 14^{+} \mathrm{CD} 16^{+}-\mathrm{M} \Phi \mathrm{s}$ may enable transition from early-activated to late-activated $\mathrm{CD}^{+} \mathrm{T}$ cells in the $\mathrm{KOA}$ joint. This hypothesis is based on the negative correlation between double-positive $\mathrm{CD} 14^{+} \mathrm{CD} 16^{+}-\mathrm{M} \Phi$ s/total SF MФs and early-activated $\mathrm{CD} 4^{+} \mathrm{T}$ cells $\left(\mathrm{CD} 9^{+}\right)$, and the positive correlation between $\mathrm{CD}_{16} 6^{+}$-MФs $\quad(89.5 \%$ being double-positive $\left.\mathrm{CD}_{14}{ }^{+} \mathrm{CD} 16^{+}-\mathrm{M} \Phi \mathrm{s}\right) /$ total SF $\mathrm{M} \Phi \mathrm{s}$ and late-activated $\mathrm{CD}^{+}{ }^{+} \mathrm{T}$ cells $\left(\mathrm{HLA}-\mathrm{DR}^{+}\right)$. Future studies may test this hypothesis, but due to the low numbers of KOA SFLs, the tests required are presently unfeasible.
While we did not focus our study on determining whether SFLs reflect synovium leukocytes, preliminary data from our laboratory (8 synovium samples vs 75 $\mathrm{SFs}$ ) show comparable overall leukocyte frequencies (Additional file 1: Figures S10, S11). Thus, while SFL populations are not fully reflective of the synovium environment, the SF is a more readily available sample for understanding the immunobiology of the joint.

Some of the limitations of our study include the exclusive use of a patient population that was in enough discomfort to seek medical treatments, thus having a limited number of patients in early stages of the disease (KL grade II, $N=20$ ) and very few at even earlier stages (KL grade I, $N=4$ ); future studies could focus on these earlier populations in which the $M \Phi$ subset ratio effects may be more pronounced. An additional limitation of our study is the fact that our samples were acquired from only one hospital, leading to a preselection of 
patients referred to it; future studies would require the use of multicentered cohorts. Patient samples were acquired from those presenting SF, excluding a subset of the KOA population with low volumes of SF, unavailable for harvest. Last, since our study was designed to determine the frequencies of SFLs and not their numbers, we were not able to simultaneously quantify absolute numbers of leukocytes, which could have allowed us to uncover other possible predictors of PROMs.

\section{Conclusions}

SF MФ subsets correlate with KOA stiffness, function and quality of life. These correlations are supportive of a biological role of $M \Phi$ subsets in KOA inflammation, which warrants further study. SF MФs may also be investigated both as biomarkers of symptomatic KOA and as targets of novel KOA therapeutics.

\section{Additional file}

Additional file 1: Supplementary Methods. Figure S1. Gating method for determining monocyte/macrophage frequencies and subpopulations ratios. Table S1. Descriptive statistics of patients with knee osteoarthritis (KOA) from whom synovial fluid (SF) was acquired. Table S2. Pain medications used. Figure S2. $\mathrm{CD} 14^{+} \mathrm{CD} 16^{+}$-monocyte/macrophages (MФs)/total SF MФs ratio vs. the use of pain medications. Figure S3. Inflammatory MФ subsets phenotype in KOA SFs and PBMCs. Figure S4. CD14+CD16+ MФs/total SF MФs at early and late stage radiographic KOA. Table S3. Effect size of ratio of MФ subsets in total SF MФs on patient reported outcome measures (PROMs) from early KOA (KL I/II) subcohort. Table S4. Effect size of ratio of MФ subsets in total SF MФs on PROMs from late KOA (KL III/IV) subcohort. Figure S5. KOA SF SCD14 and SCD163 correlation. Table S5. Effect size of SF SCD14 and SCD163 on PROMs. Figure S6. Pearson correlations between SF sCD14 vs PROMS. Figure S7. MФs and the pro-inflammatory MФs correlate with SF sCD14 levels. Figure S8. Positive correlation between SF SCD14 and CD4+ T cell frequency in SF leukocytes (SFLS). Table S6. Pearson correlations between MФs and its subsets vs SF CCL2. Table S7. Effect size of SF CCL2 on PROMs. Figure S9. Correlation between SF CD4+ T cells and SF MФs. Figure S10. Synovium leukocyte populations and SFLs. Figure S11. Synovium leukocyte and SFL MФ subsets. Figure S12. Pearson correlations between $\mathrm{CD} 14^{+} \mathrm{CD} 16^{+}$-MФs/total SF MФs vs. PROMs. (PDF $2642 \mathrm{~kb}$ )

\section{Abbreviations}

ADL: Activity of daily living; BMI: Body mass index; CCL2: Chemokine (C-C motif) ligand 2; Cl: Confidence intervals; CX3CL1: Chemokine (C-X3-C motif) ligand 1; HLA-DR: Human leukocyte antigen-antigen D related; KL: KellgrenLawrence; KOA: Knee osteoarthritis; KOOS: Knee Injury and Osteoarthritis Injury Score; MФ: Monocytes/macrophages; OA: Osteoarthritis; PBMCs: Peripheral blood mononuclear cells; PROMs: Patient-reported outcome measures; QOL: Quality of life; RA: Rheumatoid arthritis; SF: Synovial fluid; SFLs: Synovial fluid leukocytes; WOMAC: The Western Ontario and McMaster Universities Osteoarthritis Index

\section{Acknowledgements}

We would like to thank Kim Perry, Erdeta Prifty and Amanda Weston for sample management; the Arthritis Program, specially Dr. Luis Montoya and Kala Sundararajan for patient consent and patient data collection; Dr. Konstantin Shestopaloff for his guidance on statistical analysis and Prof. Mohit Kapoor and Prof. Armand Keating for sharing their laboratory space with us.

Also, we would like to thank Ahmad Kamal and Rafath Nasif for helping with processing of some of the samples.

\section{Funding}

SV received partial funding for this work from The Arthritis Society TAS-YIO 15-321. AG received partial salary support for this from The Arthritis Society TPF-15-123. Additional funding for this work was provided by the Arthritis Program's seed grant (Krembil Research Institute, UHN). We declare that there was no role of the funding source in the design of the study and collection, analysis, and interpretation of data and in writing the manuscript.

\section{Availability of data and materials}

The datasets used and/or analysed during the current study are available from the corresponding author on reasonable request.

\section{Authors' contributions}

All authors were involved in drafting the article or revising it critically for important intellectual content, and all authors approved the final version to be published. Conception and design of the study: AG and SV. Data collection and assembly: AG, RG, NNM and KWM. Statistical analysis: AG. Analysis and interpretation of data: AG, RG and SV.

\section{Ethics approval and consent to participate}

We state that the human tissues used in the study were obtained from patients, after informed consent, under the University Health Network Research Ethics Board Protocols: \#14-7483-AE.

\section{Consent for publication}

Not applicable.

\section{Competing interests}

KWM and NNM hold stock in Arthritis Innovation Corporation. There are no competing interests from the other authors.

\section{Publisher's Note}

Springer Nature remains neutral with regard to jurisdictional claims in published maps and institutional affiliations.

\section{Author details}

${ }^{1}$ Arthritis Program, University Health Network, Toronto, ON, Canada. ${ }^{2}$ Krembil Research Institute, University Health Network, Toronto, ON, Canada. ${ }^{3}$ Cell Therapy Program, University Health Network, Toronto, ON, Canada. ${ }^{4}$ Division of Orthopaedic Surgery, Toronto Western Hospital, University of Toronto, Toronto, ON, Canada. ${ }^{5}$ Institute of Biomaterials and Biomedical Engineering, University of Toronto, Toronto, ON, Canada. ${ }^{6}$ Division of Hematology, Department of Medicine, University of Toronto, Toronto, ON, Canada.

Received: 7 June 2018 Accepted: 17 December 2018 Published online: 18 January 2019

\section{References}

1. Little CB, Hunter DJ. Post-traumatic osteoarthritis: from mouse models to clinical trials. Nat Rev Rheumatol. 2013:9:485.

2. Sellam J, Berenbaum F. The role of synovitis in pathophysiology and clinical symptoms of osteoarthritis. Nat Rev Rheumatol. 2010;6:625-35.

3. Hill CL, Hunter DJ, Niu J, Clancy M, Guermazi A, Genant H, et al. Synovitis detected on magnetic resonance imaging and its relation to pain and cartilage loss in knee osteoarthritis. Ann Rheum Dis. 2007;66:1599-603.

4. de Lange-Brokaar BJE, loan-Facsinay A, Yusuf E, Kroon HM, Zuurmond A-M, Stojanovic-Susulic V, et al. Evolution of synovitis in osteoarthritic knees and its association with clinical features. Osteoarthr Cartil. 2016;24:1867-74.

5. de Lange-Brokaar BJE, loan-Facsinay A, van Osch GJVM, Zuurmond A-M, Schoones J, Toes REM, et al. Synovial inflammation, immune cells and their cytokines in osteoarthritis: a review. Osteoarthr Cartil. 2012;20:1484-99.

6. Bondeson J, Blom AB, Wainwright S, Hughes C, Caterson B, van den Berg WB. The role of synovial macrophages and macrophage-produced mediators in driving inflammatory and destructive responses in osteoarthritis. Arthritis Rheum. 2010;62:647-57.

7. Blom $A B$, van Lent $\mathrm{PL}$, Libregts $\mathrm{S}$, Holthuysen $\mathrm{AE}$, van der Kraan PM, van Rooijen $\mathrm{N}$, et al. Crucial role of macrophages in matrix metalloproteinasemediated cartilage destruction during experimental osteoarthritis: involvement of matrix metalloproteinase 3. Arthritis Rheum. 2007;56:147-57. 
8. Jónasdóttir HS, Brouwers H, Kwekkeboom JC, van der Linden HMJ, Huizinga T, Kloppenburg M, et al. Targeted lipidomics reveals activation of resolution pathways in knee osteoarthritis in humans. Osteoarthr Cartil. 2017;25:1150-60.

9. Penatti A, Facciotti F, De Matteis $R$, Larghi $P$, Paroni M, Murgo A, et al. Differences in serum and synovial CD4+ T cells and cytokine profiles to stratify patients with inflammatory osteoarthritis and rheumatoid arthritis. Arthritis Res Ther. 2017;19:103.

10. Yoo S-J, Kim J, Lee S, Yoo I-S, Shim S-C, Kwon M-H, et al. AB0796 Characterization of mononuclear cells in osteoarthritis synovial fluid monocytes. Ann Rheum Dis. 2014;73:1067.

11. Kriegova E, Manukyan G, Mikulkova Z, Gabcova G, Kudelka M, Gajdos P, et al. Gender-related differences observed among immune cells in synovial fluid in knee osteoarthritis. Osteoarthritis Cartilage. 2018;0. Available from: https://www.oarsijournal.com/article/S1063-4584(18)31233-0/abstract. [cited 2018 May 28].

12. Daghestani HN, Pieper CF, Kraus VB. Soluble macrophage biomarkers indicate inflammatory phenotypes in patients with knee osteoarthritis Arthritis Rheumatol. 2015;67:956-65

13. Stankovic A, Slavic V, Stamenkovic B, Kamenov B, Bojanovic M, Mitrovic DR. Serum and synovial fluid concentrations of CCL2 (MCP-1) chemokine in patients suffering rheumatoid arthritis and osteoarthritis reflect disease activity. Bratisl Lek Listy. 2009;110:641-6.

14. Huo LW, Ye YL, Wang GW, Ye YG. Fractalkine (CX3CL1): a biomarker reflecting symptomatic severity in patients with knee osteoarthritis. J Investig Med. 2015;63:626-31.

15. Zou Y, Li Y, Lu L, Lin Y, Liang W, Su Z, et al. Correlation of fractalkine concentrations in serum and synovial fluid with the radiographic severity of knee osteoarthritis. Ann Clin Biochem. 2013;50:571-5.

16. Li L, Jiang B-E. Serum and synovial fluid chemokine ligand 2/monocyte chemoattractant protein 1 concentrations correlates with symptomatic severity in patients with knee osteoarthritis. Ann Clin Biochem. 2015;52:276-82.

17. Wong KL, Yeap WH, Tai JJY, Ong SM, Dang TM, Wong SC. The three human monocyte subsets: implications for health and disease. Immunol Res. 2012;53:41-57.

18. Scherberich JE, Nockher WA. Blood monocyte phenotypes and soluble endotoxin receptor CD14 in systemic inflammatory diseases and patients with chronic renal failure. Nephrol Dial Transplant. 2000;15:574-8.

19. Moniuszkoa M, Bodzenta-Lukaszyka A, Kowala K, Lenczewskaa D, Dabrowskab M. Enhanced frequencies of CD14++CD16+, but not CD14 +CD16+, peripheral blood monocytes in severe asthmatic patients. Clin Immunol. 2009;130:338-46.

20. Zawada AM, Rogacev KS, Rotter B, Winter P, Marell R-R, Fliser D, et al. SuperSAGE evidence for CD14++CD16+ monocytes as a third monocyte subset. Blood. 2011;118:e50-61.

21. Yoon BR, Yoo S-J, Choi Y ho, Chung Y-H, Kim J, Yoo IS, et al. Functional Phenotype of Synovial Monocytes Modulating Inflammatory T-Cell Responses in Rheumatoid Arthritis (RA). PLOS ONE. 2014;9:e109775.

22. Smiljanovic B, Radzikowska A, Kuca-Warnawin E, Kurowska W, Grün JR, Stuhlmüller B, et al. Monocyte alterations in rheumatoid arthritis are dominated by preterm release from bone marrow and prominent triggering in the joint. Ann Rheum Dis. 2018;77:300-8.

23. Gaur P, Myles A, Misra R, Aggarwal A. Intermediate monocytes are increased in enthesitis-related arthritis, a category of juvenile idiopathic arthritis. Clin Exp Immunol. 2017;187:234.

24. Evans HG, Gullick NJ, Kelly S, Pitzalis C, Lord GM, Kirkham BW, et al. In vivo activated monocytes from the site of inflammation in humans specifically promote Th17 responses. Proc Natl Acad Sci U S A. 2009;106:6232-7.

25. Altman $\mathrm{R}$, Asch $\mathrm{E}$, Bloch D, Bole G, Borenstein D, Brandt $\mathrm{K}$, et al. Development of criteria for the classification and reporting of osteoarthritis: classification of osteoarthritis of the knee. Arthritis Rheum. 1986;29:1039-49.

26. Roos E. Knee injury and osteoarthritis outcome score. Available from: http:// www.koos.nu/index.html. [cited 2017 Dec 7].

27. Abeles RD, McPhail MJ, Sowter D, Antoniades CG, Vergis N, Vijay GKM, et al. CD14, CD16 and HLA-DR reliably identifies human monocytes and their subsets in the context of pathologically reduced HLA-DR expression by CD14hi/CD16neg monocytes: Expansion of CD14hi/CD16pos and contraction of CD14lo/CD16pos monocytes in acute liver failure. Cytometry A. 2012;81A:823-34.

28. Sainani KL. Dealing With Non-normal Data. PM\&R. 2012;4:1001-5.

29. Davison A, Hinkley D. Tests. In Bootstrap Methods and their Application (Cambridge Series in Statistical and Probabilistic Mathematics, pp. 136-190).
Cambridge: Cambridge University Press; 2017. https://doi.org/10.1017/ CBO9780511802843.005.

30. Li J, Ji L. Adjusting multiple testing in multilocus analyses using the eigenvalues of a correlation matrix. Heredity. 2005;95:221-7.

31. Pilling D, Fan T, Huang D, Kaul B, Gomer RH. Identification of markers that distinguish monocyte-derived fibrocytes from monocytes, macrophages, and fibroblasts. PLoS One. 2009;4:e7475.

32. Anderson AS, Loeser RF. Why is osteoarthritis an age-related disease? Best Pract Res Clin Rheumatol. 2010;24:15.

33. Weiss E. Knee osteoarthritis, body mass index and pain: data from the Osteoarthritis Initiative. Rheumatology. 2014;53:2095-9.

34. Elboim-Gabyzon M, Rozen N, Laufer Y. Gender differences in pain perception and functional ability in subjects with knee osteoarthritis. ISRN Orthop. 2012;2012 Available from: https://www.ncbi.nlm.nih.gov/pmc/ articles/PMC4063163/. [cited 2018 May 16].

35. van Lith M, McEwen-Smith RM, Benham AM. HLA-DP, HLA-DQ, and HLA-DR have different requirements for invariant chain and HLA-DM. J Biol Chem. 2010;285:40800-8

36. Wolk K, Döcke W-D, von Baehr V, Volk H-D, Sabat R. Impaired antigen presentation by human monocytes during endotoxin tolerance. Blood. 2000;96:218-23.

37. Miller RE, Tran PB, Das R, Ghoreishi-Haack N, Ren D, Miller RJ, et al. CCR2 chemokine receptor signaling mediates pain in experimental osteoarthritis. Proc Natl Acad Sci. 2012;109:20602-7.

38. Zarebska JM, Chanalaris A, Driscoll C, Burleigh A, Miller RE, Malfait AM, et al. CCL2 and CCR2 regulate pain-related behaviour and early gene expression in post-traumatic murine osteoarthritis but contribute little to chondropathy. Osteoarthr Cartil. 2017;25:406-12.

39. Raghu $H$, Lepus $C M$, Wang $Q$, Wong HH, Lingampalli N, Oliviero F, et al. CCL2/CCR2, but not CCL5/CCR5, mediates monocyte recruitment, inflammation and cartilage destruction in osteoarthritis. Ann Rheum Dis. 2016;2016:210426.

40. Patel AA, Zhang Y, Fullerton JN, Boelen L, Rongvaux A, Maini AA, et al. The fate and lifespan of human monocyte subsets in steady state and systemic inflammation. J Exp Med. 2017;214(7):1913-23. https://doi.org/10.1084/jem. 20170355.

\section{Ready to submit your research? Choose BMC and benefit from:}

- fast, convenient online submission

- thorough peer review by experienced researchers in your field

- rapid publication on acceptance

- support for research data, including large and complex data types

- gold Open Access which fosters wider collaboration and increased citations

- maximum visibility for your research: over $100 \mathrm{M}$ website views per year

At BMC, research is always in progress.

Learn more biomedcentral.com/submissions 